\title{
Mechanism of Probiotics in the Colon Cancer Treatment
}

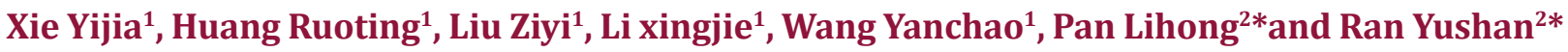 \\ ${ }^{1}$ College of Biological Sciences and Technology, Beijing Forestry University, China \\ ${ }^{2}$ State Key Laboratory of Generic Manufacture Technology of Chinese Traditional Medicine, Lunan Pharmaceutical Group Co,
} Ltd, China

*Corresponding author: Pan Lihong, State Key Laboratory of Generic Manufacture Technology of Chinese Traditional Medicine, Lunan Pharmaceutical Group Co, Ltd, China

Ran Yushan, Pan Lihong, State Key Laboratory of Generic Manufacture Technology of Chinese Traditional Medicine,

Lunan Pharmaceutical Group Co, Ltd, China

\section{ARTICLE INFO}

Received: 㓞 March 06, 2020

Published: March 16, 2020

Citation: Xie Y, Huang R, Liu Z, Li X, Ran Y, et al., Mechanism of Probiotics in the Colon Cancer Treatment. Biomed J Sci \& Tech Res 26(4)-2020. BJSTR. MS.ID.004379.

\section{ABSTRACT}

Probiotic bacteria has been found to exert anti-tumour effects against colon cancer. The possible mechanisms of probiotics in prevention and treatment of colon cancer include adjusting intestinal flora, regulating microflora metabolites, maintaining intestinal homeostasis, inducing immune regulation, antioxidant activity, inducing apoptosis and anti-inflammation.

\section{Adjust Intestinal Flora and Microflora Metabolites to Maintain Intestinal Homeostasis}

Under normal circumstances, intestinal flora constitutes the biological barrier of human intestinal tract and plays an important role in human immune regulation. The imbalance of intestinal flora may lead to colon cancerization. And the intake of specific probiotic components can improve intestinal flora, restore the balance of intestinal microorganisms, and inhibit the growth of spoilage microorganisms in the intestines [1]. Not all intestinal flora are beneficial bacteria in the human colon. Intake of certain probiotics alone can increase the number of Lactobacillus and Bifidobacterium in the fecal flora and reduce the number of pathogenic bacteria, such as Escherichia coli of colon cancer patients [1].

Firstly, probiotics can regulate $\mathrm{pH}$ and maintain intestinal homeostasis, which can prevent cancer growth. The decrease of $\mathrm{pH}$ value may directly affect the colonic epithelial cells and lead to the occurrence of colon cancer [2]. Probiotics can regulate $\mathrm{pH}$ value by reducing the reabsorption of bile acid in intestine. Probiotics can also regulate intestinal $\mathrm{pH}$ by affecting the production of shortchain fatty acids (SCFA), which is the metabolite of intestinal flora [2]. Secondly, probiotics can maintain intestinal homeostasis by inhibiting the activity of carcinogens, such as some probiotics can produce glutathione-s-transferase (GSTs) which is a main detoxification of cell anti-injury and anti-carcinogenesis [3].

Thirdly, probiotics participate in protein expression and further regulate the immune system. Probiotics play an antiinflammatory role to regulate the immune balance. About $25 \%$ of cancers are caused by chronic inflammation, such as inflammatory bowel disease leading to colon cancer (Francescone, Hou, \& Grivennikov, 2014) [4]. Clinical studies have shown that probiotics are potential in many diseases therapy, such as allergic diseases, diarrhea, inflammatory bowel disease and virus infection. Lactic acid, SCFA and other products produced by probiotics can maintain the balance between inflammatory factors (such as IL-2,IL-6 and TNF-a) and anti-inflammatory factors (such as IL-10) [5], and improve the tumour microenvironment. 
In vivo experiments showed that in the rat model of colon cancer, Bifidobacterium infantis significantly decreased the levels of proinflammatory cytokines (IL-6, IL-1 $\beta$, TNF- $\alpha$ ), and increased the activity of CD4+CD25+Foxp3+T cells (regulatory T cells) (Steidler et al., 2000) [5]. Studies on rodents found that after intragastric administration of recombinant Lactobacillus, the expression levels of anti-inflammatory substances (IL-10, human interferon- $\beta$ and antioxidants) increased. All the results proved that probiotics can improve intestinal inflammation and have cytoprotective effect.

\section{Antioxidant Activity of Probiotics}

Studies have shown that Lactococcus lactis expressing catalase can reduce the production of reactive oxygen species, reduce colon injury and inflammation, and predict tumour invasion, metastasis and proliferation [3]. Lactobacillus can produce antioxidants such as glutathione (GSH), superoxide dismutase (SOD) and catalase (CAT), and anti-angiogenic factors. Thus, it can reduce DNA damage, inhibit inflammation, control tumour size, inhibit the expression of tumour-specific proteins and polyamines, and play a role in the prevention and treatment of cancer [3]. It was found that administration of synbiotics (Lactobacillus rhamnoides, Lactobacillus acidophilus and inulin) could supress the level of malondialdehyde (MDA) and enhance the levels of glutathione reductase, superoxide dismutase and glutathione peroxidase in rats, which inhibit tumour development [6].

\section{Apoptosis Induced by Probiotics}

Probiotics have the potential to inhibit the growth and proliferation of cancer cells [3]. Chandralyer et al. [7] have shown that probiotic strains can promote the apoptosis of cancer-related cells in the hematological system. They found that Lactobacillus reuteri can enhance the ability of tumour necrosis factor (TNF) to induce apoptosis in human chronic granulocytes and leukemia cells. Lactobacillus reuteri can down-regulate the activation of nuclear factor-kappa beta (NF-kB) induced by TNF, and then inhibit cell proliferation and cell survival mediated by NF- $\kappa B$. In addition, Lactobacillus reuteri also promotes apoptosis by enhancing the activity of mitogen-activated protein kinase (MAPK). Probiotic strains can also induce apoptosis of colon cancer cells. Studies by Sara Shamekhi et al. have shown that Saccharomyce cerevisiae can induce apoptosis of colon cancer SW480 cells by regulating the Akt/ NF- $\kappa B$ signal pathway. Baldwin et al. [8] showed that Lactobacillus acidophilus and Lactobacillus casei can promote the apoptosis of colon cancer cell line (LS513). Propionibacterium freudenreichii promotes the production of reactive oxygen species by secreting SCFA, which leads to the loss of mitochondrial transmembrane potential, the activation of caspase 3 , and induces apoptosis of human colon and gastric cancer cell lines [8].

\section{Conclusion}

Many experiments have identified that probiotics play an important role in the prevention and treatment of colon cancer. It can be speculated that new prevention, diagnosis and treatment methods will appear in the future. To select functional probiotics to treat cancer, probiotics and clinical first-line drugs are combined to find the optimal strain. Combination probiotics with chemotherapeutic drugs are expected to improve the first-line drug treatment effect, so as to provide a more scientific and effective scheme for the clinical treatment of colon cancer.

\section{References}

1. Sharif M K, Mahmood S, Ahsan F (2018) Role of Probiotics Toward the Improvement of Gut Health with Special Reference to Colorectal Cancer. In Diet, Microbiome and Health p. 35-50.

2. JiaW, Xie G, Jia W (2018) Bileacid-microbiota crosstalk in gastrointestinal inflammation and carcinogenesis. Nature reviews Gastroenterology \& hepatology 15(2): 111-128.

3. Eslami M, Yousefi B, Kokhaei P, Hemati M, Nejad Z R, et al. (2019) Importance of probiotics in the prevention and treatment of colorectal cancer. Journal of cellular physiology 234(10):17127-17143.

4. Francescone R, Hou V, Grivennikov S I (2014) Microbiome, inflammation, and cancer. Cancer journal 20(3): 181-189.

5. Steidler L, Hans W, Schotte L, Neirynck S, Obermeier F, et al. (2000) Treatment of murine colitis by Lactococcus lactis secreting interleukin-10. Science 289(5483): 1352-1355.

6. Verma A, Shukla G (2014) Synbiotic (Lactobacillus rhamnosus+ Lactobacillus acidophilus+ inulin) attenuates oxidative stress and colonic damage in 1, 2 dimethylhydrazine dihydrochloride-induced colon carcinogenesis in Sprague-Dawley rats: a long-term study. European Journal of Cancer Prevention 23(6): 550-559.

7. Iyer C, Kosters A, Sethi G, Kunnumakkara A B, Aggarwal B B, et al. (2008) Probiotic Lactobacillus reuteri promotes TNF-induced apoptosis in human myeloid leukemia-derived cells by modulation of NF-kappaB and MAPK signalling. Cellular microbiology 10(7): 1442-1452.

8. Baldwin C, Millette M, Oth D, Ruiz M T, Luquet F M, et al. (2010) Probiotic Lactobacillus acidophilus and L. casei mix sensitize colorectal tumoral cells to 5-fluorouracil-induced apoptosis. Nutr Cancer 62(3): 371-378.

9. Jan G, Belzacq A, Haouzi D, Rouault A, Metivier D, et al. (2002) Propionibacteria induce apoptosis of colorectal carcinoma cells via short-chain fatty acids acting on mitochondria. Cell death and differentiation 9(2): 179-188. 
ISSN: 2574-1241

DOI: $10.26717 /$ BJSTR.2020.26.004379

Ran Yushan. Biomed J Sci \& Tech Res

(c) (P) This work is licensed under Creative

Submission Link: https://biomedres.us/submit-manuscript.php

$\begin{array}{ll}\text { BIOMEDICAL } & \text { Assets of Publishing with us } \\ \text { RESEARCHES } & \text { - Global archiving of articles } \\ \text { - Immediate, unrestricted online access } & \text { - Rigorous Peer Review Process } \\ & \text { - Authors Retain Copyrights } \\ & \end{array}$

